\title{
Fragmentarisches Strafrecht in einer global vernetzten Welt? - Bericht über die Vorträge und Diskussionen der 34. Strafrechtslehrertagung
}

\author{
Susanne Beck/Brian Valerius"
}

Die 34. Strafrechtslehrertagung (23. bis 26. Juni 2011 in Leipzig) widmete sich einer bis heute ungeklärten Grundsatzfrage: der Fragmentarietät des Strafrechts. Die Suche nach Antworten erfolgte sowohl aus theoretischem Blickwinkel als auch unter Einbeziehung des Computer- und Internetstrafrechts als aktuellem Beispiel.

Viele Fragen, die im Laufe der Tagung beleuchtet wurden, warf bereits die Bundesministerin der Justiz Sabine Leutheusser-Schnarrenberger in einer kurzen Einführung in die Thematik auf. An den Beispielen des strafrechtlichen Verbots der Zwangsheirat und der Einführung des Weltrechtsprinzips für einen möglichen Qualifikationstatbestand der Genitalverstümmelung zeigte sie, dass Strafrecht nicht als allumfassendes System staatlicher Alltagsfürsorge betrachtet oder gar als Symbol eingesetzt werden dürfe. Das ultima-ratio-Prinzip ließe Lücken im Strafrecht geradezu geboten erscheinen. Die Popularität des Strafrechts entspreche nicht seiner tatsächlichen Problemlösungskompetenz. Dem Bedürfnis, in einer individualisierten Gesellschaft durch Strafgesetze moralische Verbindlichkeit zu schaffen, dürfe nicht in jedem Fall nachgegeben werden. Zudem beklagte die Ministerin die Entformalisierung des Strafprozesses, die dessen Akzeptanz in der Öffentlichkeit verringere. Ihre abschließende Stellungnahme fand im Publikum Zustimmung: Es ist in der modernen Gesellschaft nicht einfach, den Strom gesellschaftlicher Missstände ohne Rückgriff auf das Strafrecht in gerade Bahnen zu lenken. Gerade deshalb bedarf es aber einer rationalen Kriminalpolitik und rechtspolitisch engagierten Strafrechtswissenschaft.

Die ersten beiden Referate befassten sich mit den Grundlagen des Strafrechts. „Fragmentarisches Strafrecht in Geschichte und Dogmatik "war das Thema von Professor Dr. Dr. Thomas Vormbaum (Hagen). Zur Begriffsbestimmung schickte er voraus: Strafgesetze erfassen wie jede Norm nur einen Ausschnitt der Wirklichkeit (äußere Fragmentarietät). Beschreibt eine Strafnorm nicht den ganzen vom Gesetzgeber gemeinten Sachverhalt, liegt innere Fragmentarietät vor. Deskriptive Fragmentarietät bedeutet, dass Strafrecht (seiner Natur nach) unvollständig ist. Präskriptiv ist dagegen die Forderung, Strafrecht solle fragmentarisch sein.

Die Hoffnung, Strafgesetze mögen „Inseln im Meer der Freiheit“ (Palazzo) bleiben, wurde, so Vormbaum, nicht immer geteilt: Binding forderte gerade die Beseitigung des „fragmentarischen Charakter des Strafrechts“, u.a. durch Aufhebung des Ana-

* Dr. Susanne Beck, LL.M. (LSE) ist Wissenschaftliche Mitarbeiterin am Lehrstuhl für Strafrecht, Strafprozessrecht, Rechtstheorie, Informationsrecht und Rechtsinformatik der Universität Würzburg, PD Dr. Brian Valerius ist Privatdozent an der Universität Würzburg und derzeit Lehrstuhlvertreter an der Universität Bayreuth. 
logieverbots. Im Zuge der Aufklärung (Lehre vom Gesellschaftsvertrag, Rechtsverletzungslehre nach Kant und Feuerbach) wurde Strafrecht zum Schutz der bürgerlichen Freiheit eingeschränkt. Doch schon mit Entstehung der „Rechtsgutslehre“ (Birnbaum) setzte eine Strafrechtsexpansion ein, da ein Rechtsgut für jede Strafnorm konstruierbar sei. Auch von Liszt und der Neukantianismus förderten die Strafrechtsausweitung. Dem Naturrecht der frühen Bundesrepublik folgte eine Blütezeit des Rechtsgutsbegriffs. Heute sei die Rechtsgutsdebatte von Divergenz geprägt: Teilweise wird als zentrales Rechtsgut die „Rechtsgeltung “ angesehen, teilweise werden nur personale Rechtsgüter anerkannt, teilweise wird behauptet, jegliche Definitionsversuche seien zum Scheitern verurteilt bzw. der Begriff habe keine normative Kraft. Die Abkehr vom (normativen) Rechtsgutsbegriff führte zur Orientierung an der anglo-amerikanischen Debatte (Harm Principle), der Verfassung oder zur Fokussierung auf das Verhältnismäßigkeitsprinzip.

Die Entwicklung der Gesetzgebung spiegelt nach Vormbaum die schwindende normative Kraft des Rechtsgutsbegriffs wider: seit den 1870er Jahren wächst das Strafrecht stetig. Ähnliches gelte für die Rechtsprechung: das weite Verständnis der NSZeit von zulässiger, nicht-analoger Auslegung besteht bis heute. Die Verfassungsrechtsprechung belässt dem Strafgesetzgeber erhebliche inhaltliche und formale Freiheiten. Dass politische Ziele vermehrt im Strafrecht umgesetzt werden, erklärt sich aus Sicht des Referenten u.a. mit den Großgefahren moderner Technologien, dem Bedürfnis nach einem Interventionsstaat und der Auflösung sozialer Bindungen.

Wie also ist die Strafwürdigkeit von Verhalten zu bestimmen? Vormbaum näherte sich dieser Frage von zwei Seiten. Für das Zustandekommen von Strafgesetzen fordert er eine verfassungsändernde Parlamentsmehrheit, damit die Entscheidung den gesellschaftlichen Konsens hinreichend erfasse. Die inhaltliche Schrankensetzung solle „operativ“ erfolgen. Die Grenze zwischen rein moralisch verwerflichem und strafwürdigem Verhalten sei mit dem materiellen Verbrechensbegriff zu ziehen (orientiert am Rechtsgüterschutz). Gerechtigkeit, Rechtssicherheit und Zweckmäßigkeit spielten hierbei eine wesentliche Rolle, aber auch Akzessorietät und Subsidiarität des Strafrechts. Wer Strafwünsche äußere, trage im dialektischen Verfahren die Beweislast für das Vorliegen dieser Kriterien. Auch an die Strafrechtsanwendung stellt der Referent Forderungen: Bei der teleologischen Auslegung sollte ein einziges Rechtsgut im Vordergrund stehen. Zurückhaltung sei bei systematischer Auslegung geboten, da sie eine Tendenz zur Lückenfüllung aufweise. Dieses operative Vorgehen erachtet Vormbaum als essentielles Handwerkszeug einer „Strafrechtsbegrenzungswissenschaft".

Die Notwendigkeit der Strafrechtsbegrenzung wurde in der Diskussion kaum bestritten. Gewisse Uneinigkeit bestand über die historische Herleitung (Frommel), das Ausmaß und die Ursachen der Strafrechtsexpansion - so betonte Kublen, sie sei Ne- 
benerscheinung der gesamtgesellschaftlichen Verrechtlichung und Europäisierung. Streng sah gerade die vergleichsweise Harmlosigkeit des aktuellen Strafrechts als möglichen Grund der Expansion, da mit Strafe nicht mehr notwendig gesellschaftliche Ausgrenzung verbunden sei. Umstritten war insbesondere die Auswahl der Strafwürdigkeitskriterien. Dies sei, so Weber, nicht zuletzt deshalb schwierig, weil sich das BVerfG bisher, bis auf wenige Ausnahmen, der Bestimmung derartiger Kriterien enthielt. Hilgendorf führte dies darauf zurück, dass die Verfassung dem Strafgesetzgeber eben einen weiten Handlungsspielraum eröffne und der Rechtsgutsbegriff verfassungsrechtlich nicht anschlussfähig sei (vgl. die Inzestentscheidung). Diese eher positive Einschätzung des Inzesturteils wurde nicht von allen geteilt - Paeffgen bezeichnete es als das „unerträglichste Urteil seit 60 Jahren“. Der Referent führte diesbezüglich die Relevanz des Art. 2 GG für das Strafrecht an. Die Bedeutung eines normativen Rechtsgutsbegriffs ergebe sich aus seinem Bezug zum Bürger; dies sei Kern eines freiheitlich rechtsstaatlichen Strafrechts und, so Lüderssen, eine zuverlässige Absicherung gegen eine Entpersonalisierung der Strafgesetze. Köhler dagegen betrachtete den Rechtsgutsbegriff aufgrund seiner Undeutlichkeit als historisch erledigt - es seien neue Kriterien erforderlich. Einen möglichen Konsens formulierte der Referent: Die unübersichtliche Debatte zum Rechtsgutsbegriff sei für das BVerfG tatsächlich schwer greifbar. Eine überzeugende Kriterienentwicklung sei nur in Kooperation mit Staats- und Verfassungsrechtlern möglich.

Professor Dr. Rainer Zaczyk (Bonn) behandelte „[d]ie Notwendigkeit des systematischen Strafrechts - zugleich zum Begriff ,fragmentarisches Strafrecht ““. Die heutige Begriffsverwendung „fragmentarische Natur des Strafrechts“ sah er kritisch, da sie eine vorgegebene Qualität suggeriere. Gerade als normative Orientierung sei der Begriff wegen eines Elements von Zufälligkeit ungeeignet. Sinnvoller schien ihm systematisches Vorgehen. Er setzte mit seiner Analyse bei dem Moment an, in dem ein Verurteilter seine Strafe antritt und vom Vollzugsbeamten in seine Zelle eingeschlossen wird. In diesem Endpunkt des Straf-, Strafprozess- und Strafvollzugsrechts werde das theoretische Objekt der Strafrechtswissenschaft zur Realität. Dem Verurteilten dürfe kein Unrecht geschehen, aber auch die Handlung des Vollzugsbeamten müsse als rechtmäßig begründbar sein. Dies sollte mit Blick auf die dem neuzeitlichen Verständnis vom Subjekt und seinem Recht zugrunde liegenden Vorstellungen von Staat und Freiheit erfolgen. Er präferierte insofern die von Locke, Rousseau oder Kant geprägte Vorstellung einer vorstaatlichen Freiheit vor der Hobbes'schen Ansicht, wonach der Einzelne seinen Rechtsstatus erst durch den Staat erhält. Gegenüber dem zufälligen Fragment als Begleiterscheinung der Moderne, in der fragmentarisches (vorgeblich bescheideneres) Begründen Vorrang vor theoretischen „Großsystemen“ hat, die als Hürden für die Freiheit des Denkens und wirklichkeitsfremd kritisiert werden, blieb der Referent skeptisch. Modernes Denken, das wie ein „Schmetterling von Gedankenblüte zu Gedankenblüte fliege“, scheine zwar Demokratie und Plura- 
lismus zu entsprechen, doch gebe es Bereiche, etwa das (Straf-)Recht, die das Bemühen um ein System erzwingen würden. Nur auf diese Weise könne nachvollziehbar zwischen rechtmäßiger und rechtswidriger Handlung unterschieden werden (im gewählten Beispiel zwischen der Tat des Verurteilten und dem Einschluss durch den Vollzugsbeamten).

Die Befugnis zur Strafe sei nicht heteronom begründbar, sondern müsse sich widerspruchsfrei aus dem Handeln des Täters selbst ergeben. Dies erfordere die Betrachtung einer Straftat als Verletzung des staatlich garantierten Basisvertrauens zwischen Personen. Strafe sei nur als darauf folgende Manifestation und Restitution dieses Bruchs durch den Staat, dem sich beide Beteiligten vorher zum Erhalt ihrer Freiheit untergeordnet hätten, zu rechtfertigen. Davon ausgehend bezweifelte Zaczyk die Strafbefugnis der EU oder der Völkergemeinschaft, da Strafrecht eine rechtliche Einheit voraussetze, die dem Volk eine Teilhabe an der Rechtsgemeinschaft ermögliche. Diese sei bei einem Staatenbund wie der EU nicht gegeben. Im Völkerstrafrecht wird die Tatsache, dass eine nicht legitimierte Strafausübung nichts anderes als rechtswidrige Gewalt und einen Eingriff in die Staatensouveränität darstellt, deshalb auch inzwischen verstärkt diskutiert.

Alle Aspekte des Strafrechts - die Grundlagen, dogmatischen Probleme, aber auch die empirischen Auswirkungen und Gründe des Einsatzes von Strafrecht - seien also durch die Wissenschaft des Strafrechts in einen systematischen Zusammenhang zu bringen. Bei der Entwicklung von Kriterien für die Strafgesetzgebung bedingen sich nach Zaczyk Inhalt und Form gegenseitig: so sei etwa die Bestimmtheit der Normen zwingender Bestandteil eines freiheitlichen Strafrechts. Aus der Nennung eines konkreten Strafunrechts folge zwangsläufig ein in gewissem Sinne fragmentarischer Charakter.

Mit diesen Gedanken schließt sich der Kreis zum ersten Vortrag: die Referenten sind sich insofern einig, als sie deskriptiv von Fragmentarietät des Strafrechts ausgehen, die sich aus der - möglichst konkreten - sprachlichen Erfassung von Wirklichkeit ergibt. Hinsichtlich der Prämissen der Normativität divergiert dagegen das von Vormbaum vorgeschlagene operative Vorgehen deutlich von der von Zaczyk gesetzten Prämisse einer vorstaatlichen bürgerlichen Freiheit, selbst wenn sich die Ergebnisse im Einzelnen nicht erheblich voneinander unterscheiden sollten. Und auch wenn die von Vormbaum geforderte normative Fragmentarietät nicht unsystematisch sein muss, steht bei ihm die Systematisierung doch nicht im Vordergrund.

Auch in der Diskussion manifestierten sich sowohl diese Unterschiede als auch die Übereinstimmungen. So wurde der Notwendigkeit systematischen Denkens nicht nur bei der Bewertung der Strafwürdigkeit, sondern bei der Lösung jeder Rechtsfrage (Puppe) grundsätzlich zugestimmt, wenngleich Kindhäuser und Bung bezweifelten, 
dass man mit Systematik allein in jedem (praktischen) Fall zu eindeutigen Ergebnissen komme, und Frommel hinterfragte, ob es hierzu tatsächlich hegelianischer Großsysteme bedürfe. Zur Vermeidung begrifflicher Unklarheiten regte Hörnle an, von „möglichst eingriffsarmem“ oder „freiheitsschützendem“ Strafrecht zu sprechen. Die beiden Referenten hätten sich nicht etwa widersprochen, sondern die Fragmentarietät des Strafrechts auf unterschiedlichen Ebenen diskutiert, stellte Hassemer fest: während Zaczyk den Gegensatz systematisch - unsystematisch betrachtet hätte, hätte Vormbaum den Gegensatz fragmentarisch - überfüllt diskutiert. Tatsächlich liegt Zaczyks Interesse nicht in der „Zähmung eines ungezähmten Strafrechts“, sondern in dem Bilden eines Systems, damit Strafrecht nicht bloße Gewalt darstelle. Dies schließe die Befassung mit inhaltlichen Kriterien nicht aus (Puppe, Köhler). Neben der weniger zentralen Begriffsfrage sei somit entscheidend, ob man den Schwerpunkt auf die systematisierende Orientierung an vorstaatlichen Rechten und Prinzipien lege oder ob man die Priorisierung der Freiheit als historische und damit von der Gesellschaft zu entscheidende Tatsache betrachte (Alwart). Dies zeigte sich auch an der Diskussion über die Legitimität des Europäischen und Völkerstrafrechts: je stärker die Betonung eines bestimmten Verständnisses der bürgerlichen Freiheit und des sie erhaltenden Staates, desto schwerer die Begründung dieser neuen Entwicklungen (Zaczyk insofern zustimmend Werle; skeptischer bezüglich der starken Prämissen Rosenau, Weigend). Doch bestand im Ergebnis Einigkeit, dass auch in diesen Kontexten eine Strafrechtsexpansion nicht uneingeschränkt hinzunehmen sei.

Im nationalen und grenzüberschreitenden Strafrecht ermöglicht die Argumentation innerhalb eines theoretischen Systems sicherlich widerspruchsfreiere Begründungen von Strafwürdigkeit und Strafbarkeit im Einzelfall. Für die operative Vorgehensweise spricht, dass ihre Prämissen weniger voraussetzungsreich sind. Die Grundsatzfrage der Moderne, ob und wie sich die Wirklichkeit in theoretischen Systemen abbilden lässt, wird die Rechtswissenschaft weiterhin sicher ebenso beschäftigen wie die inhaltlichen Fragen.

Mit der Wahrung des fragmentarischen Charakters des Strafrechts am konkreten Beispiel des Computer- und Internetstrafrechts beschäftigten sich die beiden nächsten Vorträge. Zunächst widmete sich Professorin Dr. Gabriele Schmölzer (Graz) unter dem Titel „Straftaten im Internet: eine materiell-rechtliche Betrachtung “ der materiellen Seite dieses Rechtsgebiets. Sie legte ihr Augenmerk darauf, Entwicklungslinien, Hintergründe und zukünftige Wege im Computer- und Internetstrafrecht aufzuzeigen. Hier ergäben sich zunehmend und mit wachsender Geschwindigkeit neue Problemstellungen, beispielsweise in sozialen Netzwerken, in denen vermehrt Angriffe auf die digitale Identität des Nutzers zu verzeichnen seien, bei dem Einsatz sog. Scareware, dem Handel mit Mitgliedsdaten, sonstigen Hacker-Angriffen und in Botnetzen. Statistiken belegten, dass einerseits trotz sinkender Gesamtkriminalität die 
Informations- und Kommunikationstechnologiekriminalität deutlich und kontinuierlich ansteige und hier andererseits die Aufklärungsrate fortwährend sinke.

Angesichts der mit diesen Entwicklungen verbundenen Herausforderungen stelle sich die Frage nach dem fragmentarischen Charakter des Strafrechts im Cyberspace. Dies betreffe nicht nur die zweckrationale Legitimation der Strafe durch fragmentarischen Rechtsgüterschutz, sondern auch eine fragmentarische Vorgehensweise, wenn Angriffshandlungen und Taterfolge benannt werden müssten und über die Sanktionierung von Vorsatz und Fahrlässigkeit oder Versuch und Vorbereitung zu entscheiden sei. Da sich die Rechts- und Sozialordnung durch den technischen Fortschritt ändere, seien zwar bestehende Straftatbestände zu ergänzen und neue zu schaffen. Jedoch dürfe das Strafrecht kein „Vehikel des Zivilrechts“ werden, d.h. nicht der Effektivierung zivilrechtlicher Rechtsdurchsetzung etwa im Urheberrecht dienen. Vielmehr bedürfe es einer „Strafwürdigkeitsprüfung lege artis“, die nicht schließungsbedürftige Strafbarkeitslücken bewusst in Kauf nehme. Prämissen einer solchen bedächtigen Vorgehensweise seien Rechtsgüterschutz, Sozialschädlichkeit und Verhältnismäßigkeit.

Dass die derzeitige Rechtslage diesen Ansprüchen nicht gerecht wird, zeigte Schmölzer in einer detaillierten Analyse der deutschen und österreichischen Computerstraftatbestände auf. Zu bemängeln bleibe in beiden Staaten die Art der Umsetzung der europäischen Vorgaben in das nationale Recht. In Österreich bildeten etwa die Strafvorschriften häufig ein komplex formuliertes Paket an objektiven und - „beweistechnisch fatal“ - subjektiven Voraussetzungen. $\$ 119$ a öStGB verkörpere sogar ein „verwirrendes Puzzle-Spiel von Tatbestandselementen “. In Deutschland wiederum begründe die deutliche Vorverlagerung der Strafbarkeit in $\$ 202$ c StGB und die damit einhergehende massive Ausweitung des Rechtsgüterschutzes eine „architektonische Schieflage“. So sei die Vorbereitung strafbar und von Amts wegen zu verfolgen, während der Versuch der vorbereiteten Antragsdelikte der $\mathbb{S} \$ 202 \mathrm{a}, 202$ b StGB nicht unter Strafe gestellt sei.

Abschließend plädierte Schmölzer dafür, dass sich das Strafrecht auch in einer global vernetzten Welt auf den Schutz der Grundwerte der Sozialordnung beschränken müsse. Damit das Strafrecht fragmentarisch bleibe, sei ein rechtsgutsbezogenes Grundkonzept erforderlich. Bislang fehle es an einem solchen soliden Unterbau, welcher ebenso der europäischen (siehe etwa den Vorschlag für eine Richtlinie über Angriffe auf Informationssysteme vom 30. September 2010) wie internationalen Expansion Einhalt gebieten könne.

In der Diskussion bemerkte Hilgendorf zunächst, wie leicht der Gesetzgeber derzeit neue Rechtsgüter schaffen könne. Die Referentin unterstrich, dass es endlich eines Systems auf Grundlage des Rechtsgüterschutzes bedürfe, bevor Spezialbereiche - 
Hilgendorf erwähnte insoweit exemplarisch das „Cloud Computing“ - behandelt werden könnten. Dies gelte auch für die von Gless hinterfragte Legitimation des nationalen Gesetzgebers, Verhaltensweisen im Internet zu regeln; hier kämen ggf. Ideen zu Weltrechtsprinzipien in Betracht. Puppe erwog, dass die fehlende Versuchsstrafbarkeit trotz Sanktionierung der Vorbereitungshandlungen dem Umstand geschuldet sein könne, dass auf letzteren der Schwerpunkt des kriminellen Unrechts liege, und verwies zum Vergleich auf die Strafbarkeit der Herstellung einer Urkunde in Gebrauchsabsicht. Auf die Nachfrage von Dölling, wie ein Selbstschutz der Nutzer sich technisch realisieren lasse und wie die im Internet zu beobachtende Selbstjustiz zu bewerten sei, führte Schmölzer aus, dass die Rolle des Nutzers als „Schwachstelle“ der Formen der Computerkriminalität, z.B. beim leichtfertigen Umgang mit seinen Daten, immer bedeutsamer werde. Auch die Selbstjustiz berge ein neues Gefahrenpotential. Abschließend gab Hoyer zu bedenken, dass der verzeichnete Kriminalitätsanstieg auch auf die neu eingeführten Straftatbestände zurückzuführen sein könnte. Im Anschluss an Puppe wendete er zudem ein, dass jedenfalls bei Personenidentität kein strafloses Zwischenstadium zwischen Vorbereitung und Vollendung existiere.

Im letzten Vortrag zum Tagungsthema widmete sich Professor Dr. Diethelm Klesczewski (Leipzig) der "Straftataufklärung im Internet“ und behandelte „technische Möglichkeiten und rechtliche Grenzen von strafprozessualen Ermittlungseingriffen im Internet“. Dabei grenzte er den Gegenstand seines Vortrags auf serverorientierte Zugriffe auf Inhaltsdaten ein, die sich auf dem für die Kommunikation gerade vorgesehenen Angebotsrechner befinden. Zugriffe auf Bestands- und Verkehrsdaten und clientorientierte Zugriffe auf den Zielrechner des Nutzers blieben ebenso ausgeklammert wie der grenzüberschreitende Aspekt von Ermittlungen im Internet. Klesczewski konzentrierte sich auf die Überwachung der Internet-Telefonie und den Zugriff auf E-Mails beim Provider.

Während bei der Überwachung der sog. anbietergestützten Internet-Telefonie, bei der ein Zugangsanbieter den Datenaustausch ermögliche, $\mathbb{\$} 100$ a StPO einschlägig sei, erwiesen sich Ermittlungen in nutzerbasierten Diensten wie Skype, die zur Datenübermittlung auf die Rechner anderer Nutzer zurückgreifen und daher die Daten in der Regel verschlüsseln, als problematisch. Die Ermittlungsbehörden infiltrierten hier bevorzugt den Rechner des Absenders oder des Empfängers, um am Endgerät als Quelle auf die unverschlüsselte Telekommunikation zuzugreifen (sog. QuellenTelekommunikationsüberwachung oder Quellen-TKÜ). Entgegen der h.A. seien solche Ermittlungen nicht durch $\mathbb{S} \mathbb{S} 100$ a f. StPO zu legitimieren, da die Vorschrift auf den Zugriff auf das Leitungsnetz ausgerichtet sei und die Quellen-TKÜ einem heimlichen Zugriff wie bei der Online-Durchsuchung gleich komme. Die strafprozessuale Quellen-TKÜ sei demnach de lege lata unzulässig. 
Unter welchen Voraussetzungen die Quellen-TKÜ de lege ferenda zu rechtfertigen sei, bestimme sich nach den Anforderungen, die das BVerfG an die Legitimation von Eingriffen in das Grundrecht auf Gewährleistung der Vertraulichkeit und Integrität informationstechnischer Systeme stelle. Bei der erforderlichen Güterabwägung des BVerfG im Einzelfall bemängelte Klesczewski eine Umkehr der Argumentationslast: Sei ein Gesetz erforderlich, um einen legitimen Zweck zu verfolgen, werde ein Verstoß gegen die Verfassung erst angenommen, wenn das betroffene Grundrecht wesentlich schwerer wiege als die Zielsetzung des Gesetzgebers. „Die Verhältnismäßigkeit verkümmer[e] zu einem bloßen Übermaßverbot.“ Außerdem werde vernachlässigt, dass im Strafprozess der Beschuldigte kein Störer wie im Recht der Gefahrenabwehr sei, sondern Verfahrensbeteiligter. Ihm gegenüber als Prozesssubjekt dürften daher grundsätzlich keine weiter reichenden Zwangsmaßnahmen als gegenüber nichtbeschuldigten Dritten (z.B. sonstigen Beteiligten am Datentransfer) gestattet werden. Zudem bleibe auch bei heimlichen Maßnahmen das Recht zu beachten, nicht aktiv an seiner Überführung mitwirken zu müssen. Äußerungen zu der dem Beschuldigten vorgeworfenen Tat zählten daher zum unantastbaren Kernbereich privater Lebensgestaltung und unterfielen einem Aufzeichnungs- und Verwertungsverbot.

Die Sicherstellung von E-Mails auf dem Server des Mail-Providers richtet sich unstreitig nach den $\mathbb{S} \$ 100 \mathrm{a}$. StPO, sofern sie gerade - sei es vom Absender zum Postfach des Empfängers bei dessen Mail-Provider oder von hier zum Empfänger - übermittelte Daten betrifft. Umstritten ist die Ermächtigungsgrundlage, wenn auf die im Postfach ruhenden Daten zugegriffen wird. Das BVerfG habe betont, dass Art. 10 Abs. 1 GG auch zwischengespeicherte Daten erfasse, gleichwohl einen Zugriff durch die Beschlagnahme- und Durchsuchungsvorschriften als legitimiert angesehen. Für eine solche Auslegung biete aber laut Klesczewski die „chaotische Gesetzeslage“ keine Basis. Mit einer anderen Auffassung die $\mathbb{\$} \$ 100$ a f. StPO als abschließende Regelung für die Überwachung der Telekommunikation zu erachten, lasse außer Acht, dass auf Elemente der Durchsuchung und Beschlagnahme in den Räumen des MailProviders nicht verzichtet werden könne; die Eingriffsvoraussetzungen der $\mathbb{S} \mathbb{S} 100$ a f. StPO mit den Beschlagnahme- und Durchsuchungsvorschriften zu kombinieren, widerspreche aber dem Vorbehalt des Gesetzes. Daher fehle auch für die Sicherstellung zwischengespeicherter E-Mails derzeit eine zureichende Ermächtigungsgrundlage. Dies gelte ebenso für einen Zugriff auf bereits gelesene, aber im Postfach beim Mail-Provider nach wie vor archivierte Daten.

Hamm und Jahn hoben in der Diskussion die Fragmentarietät des Strafprozessrechts hervor; dabei betonte Jahn, dass Ermächtigungsgrundlagen nicht vom Staat, sondern vom Bürger her zu entwickeln seien, um der Funktion der Grundrechte als Abwehrrechte gerecht zu werden. Hingegen beklagte Schünemann den „Fragmentizitätsfetischismus“ und plädierte für eine Modernisierung des Strafprozessrechts. Zustim- 
mend bemerkte der Referent, es bedürfe keines bruchstückhaften, sondern eines systematischen und die Grundrechte beachtenden Strafverfahrensrechts. Während Böse sich für die Zulässigkeit der fraglichen Ermittlungsmaßnahmen schon nach geltendem Recht aussprach, setzten sich andere mit den Anforderungen an einen Eingriff de lege ferenda auseinander und knüpften an die Schutzwürdigkeit des Kommunikationsvorgangs (Erb) bzw. an die Heimlichkeit des Zugriffs (Gleß) an. Köhler bemängelte, dass die Ermächtigungsgrundlagen nach und nach entwickelt worden seien und ein Allgemeiner Teil noch nachgeliefert werden müsse. Vogel kritisierte, dass der Vortrag die Schwerpunkte der aktuellen Diskussion ausblende, zu denen außer den bewusst ausgeklammerten Konstellationen auch die Inpflichtnahme Privater, vornehmlich der Provider, zur Straftataufklärung sowie die Rasterfahndung im Internet zählten. Klesczewski erachtete insoweit gerade die Vorratsdatenspeicherung als „staatlich verordnetes Gedächtnis“ als problematisch.

Schmölzer und Klesczewski zeigten in ihren Vorträgen auf, dass von einem Bewusstsein für die gebotene Fragmentarietät jedenfalls im Internetstraf- und -strafverfahrensrecht kaum gesprochen werden kann. Vor allem im materiellen Strafrecht ist schon jetzt eine erhebliche Ausweitung der Straftatbestände auf Vorfeldhandlungen zu beklagen, die sich im Einzelnen nicht als systematisch stimmig erweist. Auch bei der Bekämpfung neuer krimineller Erscheinungsformen wie der Internetkriminalität bedarf es aber - auf nationaler wie auf internationaler, vornehmlich europäischer Ebene - einer Rückbesinnung auf den fragmentarischen Charakter des Strafrechts.

Den Abschluss der 34. Strafrechtslehrertagung bildete traditionell der „heiße Sonntag“ (Schünemann), an dem Impulsreferate die Diskussion einer aktuellen rechtswissenschaftlichen Fragestellung vorbereiten. Zum Thema „Die strafrechtliche Bewältigung der Finanzkrise am Beispiel der Untreue“ hielt zunächst Professor Dr. Christian Schröder (Halle) fest, dass die Transaktionen der Banken, die zu der Finanzkrise führten, zwar grundsätzlich gestattet gewesen seien. Es handele sich dabei aber um Risikogeschäfte, die nach dem KWG abzusichern seien; demnach hätte nicht alles auf diese Art von Geschäften gesetzt werden dürfen. Es komme daher eine Strafbarkeit wegen Untreue in Betracht, da ein für das jeweilige Kreditinstitut existenzgefährdendes Risiko eingegangen wurde. Professor Dr. Wolfgang Woblers (Zürich) stimmte mit seinem Vorredner insofern überein, als das zur Finanzkrise führende Gebaren im Einzelfall strafbar war. Zwar sei das Strafrecht ungeeignet, soziale Krisen zu bewältigen; eine Aufarbeitung, in deren Rahmen einzelne Personen für ihre Teilbeiträge zur Krise strafrechtlich zur Verantwortung zu ziehen seien, ermögliche es jedoch durchaus. Es müsse also nicht entweder Zivil- oder Strafrecht, sondern könnten sowohl Zivil- als auch Strafrecht zur Anwendung gelangen. Auch RiBGH Professor Dr. Thomas Fischer (Karlsruhe/Würzburg) betonte, dass bei der Finanzkrise mitunter eine individuelle strafrechtliche Verantwortung vorliege. Es sei aber 
schwer, die „schwarzen Schafe“ auszusortieren, da viele Graustufen unterschieden

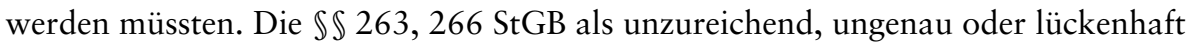
und die Motive und Ergebnisse der Finanzkrise als sozialadäquat zu erachten, sehe er kritisch. Auch nach dem Beschluss des BVerfG zur Bestimmtheit des $\mathbb{} 266 \mathrm{StGB}$ könne die erforderliche gravierende Pflichtwidrigkeit im Einzelfall anhand zahlreicher Indizien ermittelt werden.

Lüderssen zweifelte, ob die Aufarbeitung der Finanzkrise nicht doch anderen Systemen zu überlassen und das Strafrecht nur subsidiär heranzuziehen sei. Auch Streng war skeptisch, da bei einem massenweise praktizierten Verhalten kaum abschreckende Sanktionen zu erwarten seien. Schwierigkeiten bei der Strafverfolgung in der Praxis resultierten nach Jahn daraus, dass sich die prozessuale Bewältigung nach der materiellen Rechtslage richte; hier brächten aber die Anforderungen des BVerfG an die Schadensfeststellung unlösbare Probleme mit sich. Hamm berichtete in diesem Zusammenhang von einem Ermittlungsverfahren, das wegen fehlenden Vorsatzes eingestellt worden war. Zum Tatbestand der Untreue gab Kindhäuser zu bedenken, ob die Trennung zwischen Pflichtverletzung und Schaden noch aufrechtzuerhalten sei. Vogel stellte die Frage in den Raum, inwieweit bei der Schadensermittlung die Marktirrationalität zu berücksichtigen sei und ob schon die Einhaltung formaler Kriterien die Strafbarkeit ausschließen könne. Anlass zur Kritik gebe nach Puppe die Behandlung von Irrtümern z.B. in dem Mannesmann-Verfahren, da sich der Vorsatz bei gesamttatbewertenden Merkmalen nur auf die der Wertung zugrunde liegenden Tatsachen beziehe.

Wie Professor Dr. Dr. h.c. mult. Claus Roxin in seinem traditionellen Schlusswort feststellte, hatte die Tagung viele Glanzpunkte - hinsichtlich des wissenschaftlichen Ertrags, der Auswahl der Akteure, des kulturellen Angebots und des Service. Erfreulich sei, dass nunmehr nicht nur alle bedeutenden Poeten, Musiker und Wissenschaftler in Leipzig waren, sondern auch die Strafrechtslehrer. 